\title{
Reducción de la carga orgánica de aguas residuales tóxicas de los laboratorios de la unas mediante oxidación avanzada Foto - Fenton
}

\author{
Gian Marco Castillo Avila, José Antonio Blaz Matienzo
}

Universidad Nacional Agraria de la Selva, Av. Universitaria Km. 1.5 s/n, Tingo María-Perú

DOI: https://doi.org/10.33017/RevECIPeru2018.0006/

\section{Resumen}

Se investigó la reducción de la materia orgánica contenida en aguas residuales tóxicas proveniente de siete laboratorios de la Universidad Nacional Agraria de la Selva (Química General e Inorgánica, Fitoquimica, Análisis de Suelos, Bioquímica, Microscopia y Sanidad Animal) mediante el proceso Foto-Fenton solar. Para optimizar el proceso, se utilizó la metodología de superficie de respuesta, estudiando el efecto de los factores dosis de $\mathrm{H}_{2} \mathrm{O}_{2}$ y $\mathrm{Fe}$ (II) sobre la variable respuesta porcentaje de remoción de DQO. Previo al tratamiento se caracterizó el agua residual (DQO $10551 \mathrm{mgO}_{2} / \mathrm{L}, \mathrm{DBO}_{5} 850 \mathrm{mgO} / \mathrm{L}$, fenoles totales $1740 \mathrm{mg} / \mathrm{L}$, nitratos $83250 \mathrm{mg} / \mathrm{L}$, sulfatos $104402 \mathrm{mg} / \mathrm{L}$ y pH 2.8) cuyas concentraciones superan ampliamente los valores máximos admisibles. La concentración de Fe (II) fue la variable significativa sobre la remoción de la DQO. Con la optimización del proceso, se alcanzó remover un $95.3 \%$ de $\mathrm{DQO}, 67.88 \%$ de $\mathrm{DBO}_{5}, 100 \%$ de fenoles totales $91.12 \%$ de nitratos y $90.4 \%$ de sulfatos, reduciendo la concentración de los parámetros hasta valores de: $495 \mathrm{mgO}_{2} / \mathrm{L}, 273 \mathrm{mgO} / \mathrm{L}$, $0.002 \mathrm{mg} / \mathrm{L}, 7312 \mathrm{mg} / \mathrm{L}$ y $10031 \mathrm{mg} / \mathrm{L}$, respectivamente, los cuales se encuentran por debajo de los Valores Máximos Admisibles. Se logró incrementar el índice de biodegradabilidad ( $\mathrm{DBO}_{5} / \mathrm{DQO}$ ), partiendo de 0.08 de contenido de materia orgánica difícilmente biodegradable, hasta un óptimo de 0.57 de contenido de materia orgánica parcialmente biodegradable. Con lo que se comprueba que el agua residual tratada es menos toxica que el agua residual inicial.

Descriptores: Aguas residuales, Foto-Fenton, Valores máximos admisible, biodegradabilidad.

\section{Abstract}

The reduction of the organic matter content in toxic waste water from the seven laboratories at the Universidad Nacional Agraria de la Selva (General Chemistry and Inorganic, Phytochemistry, Soil Analysis, Biochemistry, Microscopy and Animal Health) through the solar photo-Fenton process was researched. To optimize the process, the response surface methods were used, studying the dosing factors of $\mathrm{H}_{2} \mathrm{O}_{2}$ and $\mathrm{Fe}$ (II) on the variable, percentage of removal response of DQO (acronym in Spanish). Before treatment, the waste water was characterized (DQO $10551 \mathrm{mgO}_{2} / \mathrm{L}, \mathrm{DBO}_{5} 850 \mathrm{mgO}_{2} / \mathrm{L}$, total phenols $1740 \mathrm{mg} / \mathrm{L}$, nitrates $83250 \mathrm{mg} / \mathrm{L}$, sulfates $104402 \mathrm{mg} / \mathrm{L}$ and $\mathrm{pH}$ 2.8); having concentrations which greatly surpassed the maximum allowable values. The concentration of Fe (II) was the significant variable for the removal of the DQO. With the optimization of the process, it was possible to remove a $95.3 \%$ of the DQO, $67.88 \%$ of the $\mathrm{DBO}_{5}$ (acronym in Spanish), $100 \%$ of the total phenols, $91.12 \%$ of the nitrates and $90.4 \%$ of the sulfates was achieved, reducing the concentration of the parameters to values of: $495 \mathrm{mgO}_{2} / \mathrm{L}, 273 \mathrm{mgO}_{2} / \mathrm{L}, 0.002 \mathrm{mg} / \mathrm{L}, 7312 \mathrm{mg} / \mathrm{L}$ and $10031 \mathrm{mg} / \mathrm{L}$, respectively, which come in under the maximum allowable values.

An increase in the biodegradable index $\left(\mathrm{DBO}_{5} / \mathrm{DQO}\right)$ was achieved, having started with 0.08 difficult biodegradable organic matter content, reaching an optimal 0.57 partially biodegradable organic matter content. With which, it is proven that the treated waste water is less toxic than the initial waste water.

Keywords: waste water, Foto-Fenton, Maximum admissible values, biodegradability. 


\section{Introducción}

Las universidades generan aguas residuales toxicas, especialmente en laboratorios de análisis químico y biológico cuya composición puede contener: indicadores, colorantes, ácidos, bases, sales, entre otras sustancias, estos desechos presentan riesgo de clase 4 (explosivos 0 inflamables sin presencia de llama, corrosivos y tóxicos) [1]. Estos residuos son producidos a diario en actividades de investigación, prácticas y extensión, y en su mayoría no son tratados [2].

La Universidad Nacional Agraria de la Selva (UNAS) no cuenta con un sistema de gestión para aguas residuales toxicas, estos residuos que se generan en los laboratorios no reciben ningún tipo de tratamiento y son descargados en el sistema de alcantarillado, lo que genera problemas a corto o largo plazo como: Bioacumulación, magnificación en la cadena trófica, entre otros problemas ambientales, situación que hace que el recurso hídrico pierda su calidad [3]. Enviar estas aguas residuales para su tratamiento a empresas prestadoras de servicio, es extremadamente caro y solo transfiere el problema de manos. Por otro lado, algunos compuestos son tóxicos para las bacterias, lo que hace inoperable un tratamiento biológico [4].

El aumento en los niveles de contaminación de las aguas superficiales, deja en la necesidad de buscar alternativa de tratamiento que sea efectivo y sostenible en el tiempo. Los procesos de oxidación avanzada (POA), se perfilan como una alternativa atractiva desde el punto de vista técnico y ambiental para la degradación de aguas residuales recalcitrantes [4] y más específicamente del proceso fenton, en el que se utiliza como reactivos al peróxido de hidrogeno y sales de $\mathrm{Fe}^{+2}$ para la formación de radicales hidroxilo, utilizando un reactor de diseño muy simple [5].

En los experimentos tipo Fenton es muy importante tener en cuenta la dosis correcta de los reactivos peróxido y catalizador. Muchos estudios aplican el enfoque de mover un factor a la vez. En la actualidad existe un creciente reconocimiento de que esta práctica debe ser sustituida por una base sólida de métodos quimiométricos basados en el diseño estadístico de experimentos. Los análisis estadísticos son más eficientes, ya que representan los efectos de la interacción entre las variables estudiadas y determinan con mayor precisión la combinación de los niveles que produce el óptimo del proceso [6].

\section{Metodología}

Se aplicó la metodología de superficie de respuesta para optimizar el proceso de oxidación avanzada con el reactivo fenton, estudiando el efecto de los factores catalizador y oxidante (concentraciónes en $\mathrm{g} / \mathrm{l}$ de $\mathrm{Fe}(\mathrm{II})$ y $\mathrm{H}_{2} \mathrm{O}_{2}$ ) respectivamente, sobre el agua residual tóxica proveniente de los diferentes laboratorios de la Universidad Nacional Agraria de la Selva (Química General e Inorgánica, Fitoquímica, Análisis de Suelos, Bioquímica, Microscopia y Sanidad Animal), utilizando como como variable respuesta la demanda química de oxígeno.

Los ensayos se realizaron en un colector solar cilíndrico parabólico compuesto. Que consiste en un módulo con dos tubos de boro silicato y láminas de aluminio. En cuanto al material reflectante del CPC fue de aluminio, el cual es el único metal que presenta reflectividades de $92,3 \%$ a $280 \mathrm{~nm}$ hasta $92,5 \%$ a 385 nm [7], además los tubos de Borosilicato fueron de un diámetro de $30 \mathrm{~mm}$ el cual tiene la sección del diámetro ideal para CPC [8]. El sistema operó de modo discontinuo (Bach) durante dos horas, con recirculación forzada, donde el flujo generado por la bomba, fue de carácter turbulento con el que se alcanza una distribución uniforme y una buena suspensión del catalizador, como recomienda [9].

En cuanto al funcionamiento, el equipo tenia la capacidad para tratar $1 \mathrm{Lt}$ de agua residual, el sistema operó de modo discontinuo (Bach) durante dos horas, con recirculación forzada, el caudal generado por la bomba fue de $0.0015 \mathrm{~m}^{3} / \mathrm{s}$, cuyo flujo fue de carácter turbulento con el que se alcanza una distribución uniforme y una buena suspensión del catalizador, como recomienda [9]. En cuanto al funcionamiento, se homogeniza el agua residual con la dosis de $\mathrm{Fe}$ (II) (sulfato ferroso $\mathrm{FeSO}_{4}$ ), seguidamente se abre la llave 1 , a continuación se abre la llave 2 para dar paso a la dosis de $\mathrm{H}_{2} \mathrm{O}_{2}$ la cual ingresaba de manera continua al sistema con un caudal de $0.014 \mathrm{ml} / \mathrm{s}$. El objetivo de inyectar el peróxido de manera constante es para minimizar las pérdidas de radical hidroxilo, ya que el tiempo de vida media del radical hidroxilo es de sólo microsegundos, por tanto, debe ser generado constantemente en el medio [10]. Concluido el tiempo de retención hidráulica (2 horas) se abre la llave 3 para recoger la muestra tratada. Cabe mencionar que la llave número 1 no se debe cerrar para que el $\mathrm{CO}_{2}$ generado en las reacciones pueda salir y así evitar un colapso del sistema. El esquema de funcionamiento del equipo se muestra a continuación en la figura 1 : 
Julio 2018

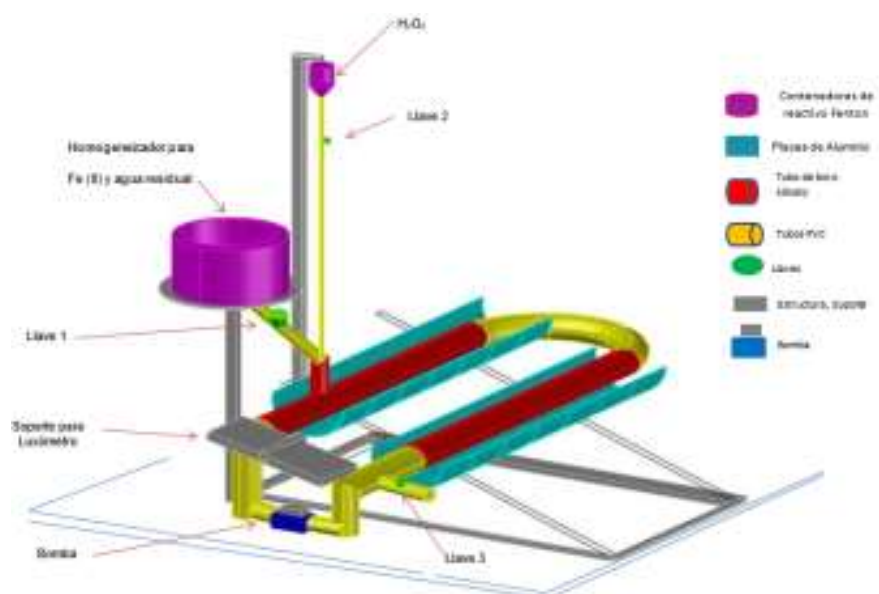

Figura 1. Estructura del equipo Fotocatalizador.

\subsection{Determinación de los parámetros fisicoquímicos de las aguas residuales tóxicas}

\subsubsection{Caracterización de aguas residuales tóxicas}

Previo a la caracterización, se recolecto el agua residual de seis laboratorios (Química General e Inorgánica, Fotoquímica, Análisis de Suelos, Bioquímica, Microscopia y Sanidad Animal), formando de esta manera una muestra compuesta como recomienda [4]. La recolección se llevó a cabo durante el periodo de clases, el sistema de recolección fue selectivo es decir se priorizó la recolección de los aguas residuales en cada práctica de laboratorio

La caracterización del agua residual tóxica, se realizó en base a los métodos estandarizados, [11] y [12] en términos de: DQO (METODO 5220 D), Fenoles (5530 B, C), Nitratos y Sulfatos (EPA 300.1), DBO 5 (METODO 5210 B), Oxidabilidad al Permanganato (norma española UNE-EN ISO 8467 elaborado por el comité técnico AEN/CTN77 [13]) y $\mathrm{pH}$ (METODO 4500-H+B). El agua residual tratada con la dosis óptima fue caracterizada en base a los mismos parámetros evaluados en el agua residual inicial.

\subsection{Optimización del proceso Foto-Fenton mediante la metodología de superficie de respuesta}

Para la optimización del proceso foto-fenton sobre las aguas residuales de los laboratorios de la UNAS, se aplicó la metodología de superficie de respuesta (MSR), estudiando el efecto de los factores (dosis en $\mathrm{g} / \mathrm{L}$ de $\mathrm{Fe}(\mathrm{II})$ y $\mathrm{H}_{2} \mathrm{O}_{2}$ ) sobre la variable respuesta
DQO expresada como Oxidabilidad al permanganato, Como se detalla a continuación:

\subsubsection{Pruebas preliminares}

Para el inicio de los experimentos se ubicó una dosis de partida la cual fue $9 \mathrm{~g} / \mathrm{L}$ de $\mathrm{Fe}$ (II) y de 220.7 $\mathrm{g} / \mathrm{L}$ de $\mathrm{H}_{2} \mathrm{O}_{2}$ [4]. Con esta dosis de partida, se realizaron pruebas preliminares en un diseño $3^{\mathrm{k}}$ factores siendo $\mathrm{K}$ el número de variables $(\mathrm{K}=2)$, probado en 3 niveles (alto, medio y bajo), para el $\mathrm{H}_{2} \mathrm{O}_{2}(120,180$ y 220$) \mathrm{g} / \mathrm{L}$ y para el Fe II $(3.5,6$ y 8.5$)$ $\mathrm{g} / \mathrm{L}$, se realizaron entonces nueve experimentos. Mediante los resultados obtenidos, se procedió a descartar los niveles que presentaron menor remoción de DQO y se realizaron nuevos experimentos para ajustar un modelo de primer orden.

\subsubsection{Experimento con modelo de primer orden}

El diseño factorial utilizado fue $2^{\mathrm{k}}$, aumentando cinco puntos centrales, se realizaron entonces nueve tratamientos. El objetivo principal de esta etapa es confirmar la influencia significativa de los factores sobre la característica de calidad a optimizar, determinar la significancia de las interacciones entre dichos factores, estimar el modelo de regresión que describa el comportamiento del efecto de dichos factores y determinar en qué dirección se encuentran las condiciones más probables para optimizar el proceso [14].

\subsubsection{Método de máxima pendiente en ascenso}

El método de máxima pendiente en ascenso es un procedimiento para recorrer secuencialmente la trayectoria de la máxima pendiente, que nos lleva en dirección del máximo aumento de la respuesta [14] el modelo ajustado de primer orden es:

$$
\hat{Y}=\widehat{\beta}_{o}+\sum_{i=1}^{K} \widehat{\beta}_{i} X_{i}(1) \text {. }
$$

Los incrementos a lo largo de la trayectoria son proporcionales a los coeficientes de regresión ( $\mathrm{B}_{0}$, $\mathrm{B}_{1}, \mathrm{~B}_{2}$ ), el tamaño real del paso, lo determina el experimentador en base a su experiencia [14]. Los experimentos se llevaron a cabo hasta que se se ajustó un nuevo modelo de primer orden.

Algoritmo para determinar las coordenadas de un punto en la trayectoria de máxima pendiente en ascenso: Un algoritmo propuesto por [14] es el siguiente: observó un decremento en la respuesta, entonces 
Supóngase que el punto $x_{1}=x_{2}=\ldots=x_{k}=0$. Se elige un tamaño de incremento o "escalón" en una de las variables del proceso, digamos $\Delta x j$, usualmente se elige la variable de la que más se sabe, o la que tiene el Mayor coeficiente de regresión absoluto $\left|B_{j}\right|$; El tamaño de incremento en las otras variables es:

$$
\Delta \mathrm{X}_{\mathrm{I}}=\frac{\mathrm{B}_{\mathrm{i}}}{\mathrm{B}_{\mathrm{j}} / \Delta \mathrm{X}_{\mathrm{j}}}
$$

Dónde: $i=1,2 \ldots, K \neq j$. Se convierte $\Delta X_{I}$, de variables codificadas a variables naturales. Identificado la región experimental, se procede a realizar experimentos con segundo orden hasta alcanzar el óptimo local.

\subsubsection{Experimento con modelo de segundo orden}

Al encontrarse cerca del óptimo, es necesario ajustar un modelo de segundo orden, el objetivo primordial de esta etapa es modelar el comportamiento del proceso en una región muy precisa y relativamente pequeña. [14].

El modelo ajustado de una superficie de respuesta es:

$$
\hat{Y}=\widehat{\beta}_{o}+\sum_{i=1}^{K} \widehat{\beta}_{i} X_{i}+\sum_{i} \sum_{j} \widehat{\beta}_{i j} X_{i} X_{j} \quad \text { i<j }(3) \text {. }
$$

Dónde: $\hat{Y}$ es el valor de la respuesta esperada, $\widehat{\beta}_{0}$ $\widehat{\beta}_{i}, \widehat{\beta}_{\mathrm{ii}}$, parámetros del modelo, $X_{i}$ y $X_{j}$ son los códigos de los factores estudiados y $k$ es el número de factores analizados. Se usó un Diseño central compuesto tipo estrella rotable para ajustar la superficie de respuesta.

Se utilizó un diseño central compuesto (CCD) en K factores requiere $2^{k}$ corridas factoriales, $2^{k}$ corridas axiales y al menos un punto central (en el presente estudio se usaron 5 puntos centrales).

Técnica de optimización: Una vez obtenido el modelo debidamente ajustado y validado, se utilizó el análisis canónico, el software realiza los cálculos para maximizar la respuesta una vez calculada la dosis óptima predicha por el software en variables codificadas, se procede a convertir en variable natural, según la ecuación:

$$
X i=\frac{\left(A_{i}-\bar{A}\right)}{D}
$$

Dónde: $A i$ es el $i$-ecimo nivel del factor, $\bar{A}$ es el nivel promedio del factor $A$ y $D=\frac{1}{2}\left(A_{2}-A_{1}\right)$. Al aplicar la ecuación (4) se obtienen las concentraciones reales en $\mathrm{g} / \mathrm{L}$ del catalizador y oxidante, con la dosis óptima calculada se realizó un nuevo experimento, y se evalúo el porcentaje de remoción de los parámetros evaluados inicialmente.

\subsection{Cálculo de la biodegradabilidad}

Para determinar la biodegradabilidad, se utilizan los parámetros tales como $\mathrm{DQO}_{\text {y }} \mathrm{DBO}_{5}$ [15]. La relación de $\left(\mathrm{DBO}_{5} / \mathrm{DQO}\right)$ se emplea como parámetro indicador de la mejora de la biodegradabilidad, este indicador se calcula de la siguiente manera:

$$
\text { Biod }=\frac{D B O_{5}}{D Q O}
$$

Dónde: Biod: Biodegradabilidad; $\mathrm{DBO}_{5}$ : Demanda Bioquímica de Oxígeno en 5 días; DQO:Demanda Quimica de Oxigeno. Esta relación indica que fracción de la materia orgánica presente en el agua residual es susceptible de ser descompuesta biológicamente [16].

\section{Contenido de la sección de resultados}

\subsection{Determinación de los parámetros químicos en aguas residuales tóxicas de los laboratorios de la UNAS y su comparación con los valores máximos admisibles}

Se presentan las concentraciones de los parámetros químicos (DQO, $\mathrm{DBO}_{5}$, fenoles totales,

\begin{tabular}{|c|c|c|}
\hline Parámetro & $\begin{array}{l}\text { Agua residual } \\
\text { original }\end{array}$ & $\underset{1}{\text { VMA }}$ \\
\hline $\begin{array}{l}\text { Demanda química de } \\
\text { oxigeno }\left(\mathrm{mgO}_{2} / \mathrm{L}\right)^{*}\end{array}$ & 10550 & 1000 \\
\hline $\mathrm{DBO}_{5}\left(\mathrm{mgO}_{2} / \mathrm{L}\right)$ & 850 & 500 \\
\hline $\mathrm{pH}$ & 2.8 & $6-9$ \\
\hline $\begin{array}{c}\text { Fenoles totales* } \\
\mathrm{mg} / \mathrm{L})\end{array}$ & 1740 & NA \\
\hline $\operatorname{Nitratos}^{*}(\mathrm{mg} / \mathrm{L})$ & 83250 & NA \\
\hline Sulfatos $^{\star}(\mathrm{mg} / \mathrm{L})$ & 104402 & 500 \\
\hline
\end{tabular}
sulfatos, nitratos y $\mathrm{pH}$ ) y su comparación con los Valores Máximos Admisibles, así se tiene:

Tabla 1. Caracterización del agua residual inicial.

${ }^{1}$ VMA valores máximo admisibles para la descarga de aguas residuales no domesticas al sistema de alcantarillado.

De la caracterización del agua residual tóxica en la tabla 1, se puede observar que la concentración de los parámetros evaluados, superan ampliamente los Valores Máximos Admisibles de la normativa ambiental peruana. 


\subsection{Optimización del proceso Foto-Fenton a través de la metodología de superficie de respuesta}

Se realizaron pruebas preliminares, probado en tres niveles alto, medio y bajo, partiendo con las dosis de $\mathrm{H}_{2} \mathrm{O}_{2}(220,180$ y 120 ,) g/L y para Fe (II) $(16,9$ y 2) $\mathrm{g} / \mathrm{L}$, utilizando un diseño factorial $3^{2}$. De los resultados en las pruebas preliminares, se obtuvo que con las dosis de $(180,2)$ y $(120,9) \mathrm{g} / \mathrm{L}$ de $\mathrm{H}_{2} \mathrm{O}_{2}$ y $\mathrm{Fe}$ (II), se alcanza remover un $85.20 \%$ y $80.90 \%$ de materia orgánica respectivamente, por consiguiente, se realizaron nuevos experimentos próximos a esta región de experimentación. Los nuevos experimentos fueron ajustados a un modelo de primer orden, se utilizó un diseño factorial $2^{2}$ aumentando en cinco puntos centrales, como se detalla a continuación en la tabla 2:

Tabla 2. Pruebas preliminares para determinar la dosis apropiada de $\mathrm{H}_{2} \mathrm{O}_{2}$ y $\mathrm{Fe}$ (II).

\begin{tabular}{|c|c|c|c|c|}
\hline \multicolumn{2}{c|}{$\begin{array}{c}\text { Variables } \\
\text { Naturales }\end{array}$} & \multicolumn{2}{c|}{$\begin{array}{c}\text { Variables } \\
\text { codificadas }\end{array}$} & \multirow{2}{*}{$\begin{array}{c}\text { Remoción } \\
\text { DQO(\%) }\end{array}$} \\
\cline { 1 - 3 }$£_{1}$ & $£_{2}$ & $\mathrm{X}_{1}$ & $\mathrm{X}_{2}$ & \\
\hline 120 & 2 & -1 & -1 & $66.08 \%$ \\
\hline 120 & 9 & -1 & 1 & $64.25 \%$ \\
\hline 180 & 2 & 1 & -1 & $85.37 \%$ \\
\hline 180 & 9 & 1 & 1 & $80.96 \%$ \\
\hline 150 & 5.5 & 0 & 0 & $74.77 \%$ \\
\hline 150 & 5.5 & 0 & 0 & $75.62 \%$ \\
\hline 150 & 5.5 & 0 & 0 & $74.86 \%$ \\
\hline 150 & 5.5 & 0 & 0 & $76.41 \%$ \\
\hline 150 & 5.5 & 0 & 0 & $75.68 \%$ \\
\hline
\end{tabular}

En la tabla 3 se muestra el análisis de varianza. Mediante el método de mínimos cuadrados se obtiene la ecuación del modelo lineal ajustado:

Remocion de $\mathrm{OP} \%=32.3403+0.30 \mathrm{H}_{2} \mathrm{O}_{2}-0.445 \mathrm{Fe}$ (II) (6)

El factor $R^{2}$ es $97.165 \%$, el valor-P en la tabla de análisis de varianza (ANVA) es menor que 0.05, indicando que existe una relación estadísticamente significativa entre las variables con un nivel de confianza del $95.0 \%$.

Por otro lado la prueba de falta de ajuste no es significativa, lo que indica que existe relación funcional entre los factores experimentales y la variable respuesta.
Tabla 3. Análisis de varianza para el modelo de primer orden.

\begin{tabular}{|c|c|c|c|c|c|}
\hline Fuente & SC & GL & CM & Fc & P-valor \\
\hline Modelo & 332.47 & 2 & $\begin{array}{c}166.2 \\
3\end{array}$ & $\begin{array}{c}138.1 \\
4\end{array}$ & $\begin{array}{c}< \\
0.0001\end{array}$ \\
\hline $\mathrm{A}: \mathrm{H}_{2} \mathrm{O}_{2}$ & 322.92 & 1 & $\begin{array}{c}322.9 \\
2\end{array}$ & $\begin{array}{c}268.3 \\
5\end{array}$ & $\begin{array}{c}< \\
0.0001\end{array}$ \\
\hline $\mathrm{B}: \mathrm{Fe}(\mathrm{II})$ & 9.55 & 1 & 9.55 & 7.93 & 0.0305 \\
\hline Residual & 7.22 & 6 & 1.2 & & \\
\hline $\begin{array}{c}\text { Falta de } \\
\text { ajuste }\end{array}$ & 5.34 & 2 & 2.67 & 5.67 & 0.068 \\
\hline $\begin{array}{c}\text { Error } \\
\text { puro }\end{array}$ & 1.88 & 4 & 0.47 & & \\
\hline Total & 339.69 & 8 & & & \\
\hline
\end{tabular}
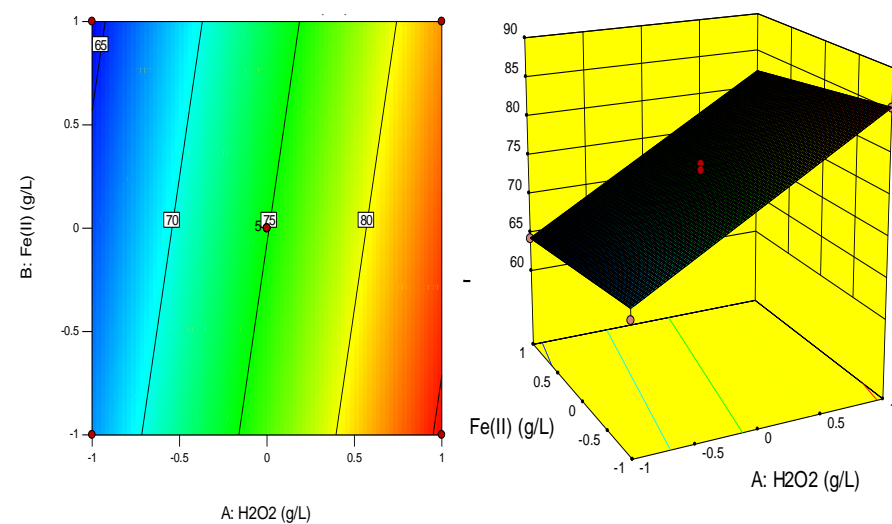

Figura 2. Gráfica de contornos y superficie de respuesta del modelo de primer orden para la remoción de $D Q O$ (\%).

Al examinar la gráfica de contornos y la ecuación (6) se observa que para alejarse del centro del diseño es necesario desplazarse 0.30 unidades en dirección de X1 por cada - 0.445 unidades en dirección de X2. Se usó $1.5 \mathrm{~g} / \mathrm{L}$ de $\mathrm{Fe}$ (II) en la concentración del catalizador en la variable natural $£_{2}$. Mediante la ecuación (2) se realiza un cambio de variable, obteniendo que la variable real $£_{1}$, equivale a $-1.011 \mathrm{~g} / \mathrm{L}$ de $\mathrm{H}_{2} \mathrm{O}_{2}$. Por lo tanto se moverá en 1.5 $\mathrm{g} / \mathrm{L}$ de $\mathrm{Fe}$ (II) y en $-1.011 \mathrm{~g} / \mathrm{L} \mathrm{H}_{2} \mathrm{O}_{2}$, como se muestra en la tabla 4.

Tabla 4. Experimento de máximo ascenso de la colina.

\begin{tabular}{|c|c|c|c|c|c|}
\hline $\begin{array}{c}\text { Increm } \\
\text { ento }\end{array}$ & \multicolumn{2}{|c|}{$\begin{array}{c}\text { Variables } \\
\text { codificadas }\end{array}$} & \multicolumn{2}{c|}{$\begin{array}{c}\text { Variables } \\
\text { Naturales }\end{array}$} & $\begin{array}{c}\text { Remoció } \\
\text { n DQO } \\
(\%)\end{array}$ \\
\cline { 2 - 5 } & $\mathrm{X}_{1}$ & $\mathrm{X}_{2}$ & $£_{1}$ & $£_{2}$ & - \\
\hline $\mathrm{O}$ & 0 & 0 & 180 & 2 & - \\
\hline
\end{tabular}




\begin{tabular}{|c|c|c|c|c|c|}
\hline$\Delta$ & -0.674 & 1 & -1.011 & 1.5 & - \\
\hline $\mathrm{O}+\Delta$ & -0.674 & 1 & 178.989 & 3.5 & $85.41 \%$ \\
\hline $\mathrm{O}+2 \Delta$ & -1.348 & 2 & 177.978 & 5 & $85.89 \%$ \\
\hline $\mathrm{O}+3 \Delta$ & -2.022 & 3 & 176.967 & 6.5 & $86.51 \%$ \\
\hline $\mathrm{O}+4 \Delta$ & -2.696 & 4 & 175.956 & 8 & $87.46 \%$ \\
\hline $\mathrm{O}+5 \Delta$ & -3.37 & 5 & 174.945 & 9.5 & $88.85 \%$ \\
\hline $\mathrm{O}+6 \Delta$ & -4.044 & 6 & 173.934 & 11 & $90.46 \%$ \\
\hline $\mathrm{O}+7 \Delta$ & -4.718 & 7 & 172.923 & 12.5 & $88.47 \%$ \\
\hline $\mathrm{O}+8 \Delta$ & -5.392 & 8 & 171.912 & 14 & $86.63 \%$ \\
\hline
\end{tabular}

\begin{tabular}{|l|c|c|c|c|c|}
\hline $\begin{array}{l}\text { Falta de } \\
\text { ajuste }\end{array}$ & 9.83 & 2 & 4.92 & $\begin{array}{c}279.9 \\
3\end{array}$ & $\begin{array}{c}< \\
0.001\end{array}$ \\
\hline $\begin{array}{c}\text { Error } \\
\text { puro }\end{array}$ & 0.07 & 4 & 0.018 & & \\
\hline Total & 10.09 & 8 & & & \\
\hline
\end{tabular}

De acuerdo a lo observado en la Tabla 6, el valor-P en la tabla ANVA es mayor que 0.05 , indicando que no existe una relación estadísticamente significativa entre las variables en estudio, además se obtiene que el factor $R^{2}$ ajustado es cero (0) lo que indica la no existencia de relación lineal estocástica, por consiguiente se realizan experimentos con un modelo de segundo orden (diseño central compuesto tipo estrella rotable):

Al observar la Tabla 4, se puede apreciar un incremento de la remoción hasta el sexto experimento, a partir del séptimo se produce un decremento de remoción.

Por lo tanto se ajusta un segundo modelo de primer orden, y la región de exploración para $\Sigma_{1}$ es $(173.934,174.945)$ y para $£_{2}$ es $(11,9.5)$. Con estas condiciones de experimentación se ajusta un nuevo modelo de primer orden:

Tabla 5. Datos para el segundo modelo de primer orden.

\begin{tabular}{|c|c|c|c|c|}
\hline \multicolumn{2}{|c|}{$\begin{array}{c}\text { Variables } \\
\text { Naturales }\end{array}$} & \multicolumn{2}{c|}{$\begin{array}{c}\text { Variables } \\
\text { codificadas }\end{array}$} & \multirow{2}{*}{$\begin{array}{c}\text { Remoción } \\
\text { DQO (\%) }\end{array}$} \\
\cline { 1 - 4 }$£_{1}$ & $£_{2}$ & X1 & X2 & \\
\hline 173.934 & 9.5 & -1 & -1 & $90.77 \%$ \\
\hline 173.934 & 11 & -1 & 1 & $89.92 \%$ \\
\hline 174.945 & 9.5 & 1 & -1 & $90.13 \%$ \\
\hline 174.945 & 11 & 1 & 1 & $91.36 \%$ \\
\hline 174.4395 & 10.25 & 0 & 0 & $92.41 \%$ \\
\hline 174.4395 & 10.25 & 0 & 0 & $92.50 \%$ \\
\hline 174.4395 & 10.25 & 0 & 0 & $92.78 \%$ \\
\hline 174.4395 & 10.25 & 0 & 0 & $92.50 \%$ \\
\hline 174.4395 & 10.25 & 0 & 0 & $92.59 \%$ \\
\hline
\end{tabular}

Se realizó el análisis de varianza para el segundo modelo de primer orden, así se tiene:

Tabla 6. Análisis de varianza para el segundo modelo de primer orden.

\begin{tabular}{|l|c|c|c|c|c|}
\hline Fuente & SC & GL & CM & Fc & p-valor \\
\hline Modelo & 0.19 & 2 & 0.095 & 0.058 & 0.944 \\
\hline $\mathrm{A}: \mathrm{H}_{2} \mathrm{O}_{2}$ & 0.16 & 1 & 0.16 & 0.097 & 0.766 \\
\hline $\mathrm{B}: \mathrm{Fe}(\mathrm{II})$ & 0.03 & 1 & 0.03 & 0.018 & 0.896 \\
\hline Residual & 9.9 & 6 & 1.65 & & \\
\hline
\end{tabular}

Tabla 7. Diseño central compuesto tipo estrella rotable.

\begin{tabular}{|c|c|c|c|c|}
\hline \multicolumn{2}{|c|}{$\begin{array}{c}\text { Variables } \\
\text { Naturales }\end{array}$} & \multicolumn{2}{c|}{$\begin{array}{c}\text { Variables } \\
\text { codificadas }\end{array}$} & $\begin{array}{c}\text { Remoció } \\
n\end{array}$ \\
\cline { 1 - 3 }$£_{1}$ & $£_{2}$ & X1 & X2 & \\
\hline 173.934 & 9.5 & -1 & -1 & $94.56 \%$ \\
\hline 173.934 & 11 & -1 & 1 & $92.45 \%$ \\
\hline 174.945 & 9.5 & 1 & -1 & $93.75 \%$ \\
\hline 174.945 & 11 & 1 & 1 & $92.71 \%$ \\
\hline 174.440 & 10.25 & 0 & 0 & $94.81 \%$ \\
\hline 174.440 & 10.25 & 0 & 0 & $94.82 \%$ \\
\hline 174.440 & 10.25 & 0 & 0 & $94.81 \%$ \\
\hline 174.440 & 10.25 & 0 & 0 & $94.83 \%$ \\
\hline 174.440 & 10.25 & 0 & 0 & $94.82 \%$ \\
\hline 174.440 & 11.31 & 0 & 1.414 & $92.61 \%$ \\
\hline 175.154 & 10.25 & 1.414 & 0 & $93.46 \%$ \\
\hline 173.725 & 10.25 & -1.414 & 0 & $91.74 \%$ \\
\hline 174.440 & 9.19 & 0 & -1.414 & $93.90 \%$ \\
\hline
\end{tabular}

Al realizar el análisis de varianza con los datos de la tabla 7 , se obtuvo un coeficiente $\mathrm{R}^{2}$ ajustado de $79.75 \%$, y el modelo:

Remocion de $\mathrm{OP} \%=+94.818+0.235 \mathrm{H}_{2} \mathrm{O}_{2}-$ $0.622 \mathrm{Fe}(\mathrm{II})+0.268 \mathrm{H}_{2} \mathrm{O}_{2} * \mathrm{Fe}(\mathrm{II})-0.999 \mathrm{H}_{2} \mathrm{O}_{2}{ }^{2}-$ $0.62 \mathrm{Fe}(I I)^{2}(7)$

En este caso los resultados no se ajustaron al modelo. Para la optimización del porcentaje de remoción es necesario ajustar un modelo que describa adecuadamente el comportamiento, por lo tanto, se decidió eliminar la condición experimental (173.725 g / $\mathrm{L} \mathrm{H}_{2} \mathrm{O}_{2}$ y $10.25 \mathrm{~g} / \mathrm{L}$ de Fe II), ya que se encuentra fuera de la región de búsqueda del mejor 
tratamiento. Los resultados del ANVA al eliminar este valor se muestra a en la Tabla 8:

Tabla 8. ANVA del modelo cuadrático ajustado, eliminando un tratamiento

\begin{tabular}{|l|c|c|c|c|c|}
\hline Fuente & SC & GL & CM & Fc & $\begin{array}{c}\text { P- } \\
\text { Valor }\end{array}$ \\
\hline Modelo & 9.77 & 5 & 1.95 & 51.02 & $\begin{array}{c}< \\
0.0001\end{array}$ \\
\hline${\mathrm{A}-\mathrm{H}_{2} \mathrm{O}_{2}}$ & 0.066 & 1 & 0.066 & 1.72 & 0.2381 \\
\hline $\mathrm{B}-\mathrm{Fe}(\mathrm{II})$ & 3.09 & 1 & 3.09 & 80.74 & 0.0001 \\
\hline $\mathrm{AB}$ & 0.29 & 1 & 0.29 & 7.47 & 0.034 \\
\hline $\mathrm{A}^{\wedge} 2$ & 1.65 & 1 & 1.65 & 42.97 & 0.0006 \\
\hline $\mathrm{B}^{\wedge} 2$ & 4.11 & 1 & 4.11 & 107.28 & $\begin{array}{c}< \\
0.0001\end{array}$ \\
\hline Residual & 0.23 & 6 & 0.038 & & \\
\hline $\begin{array}{l}\text { Falta de } \\
\text { ajuste }\end{array}$ & 0.23 & 2 & 0.11 & 1639.94 & $<$ \\
\hline $\begin{array}{l}\text { Error } \\
\text { puro }\end{array}$ & 0 & 4 & 0 & & 0.0001 \\
\hline \begin{tabular}{c} 
Total \\
\hline
\end{tabular} & 10 & 11 & & & \\
\hline
\end{tabular}

El valor-P en la tabla 8 , es menor que 0.05 , indicando que existe una relación estadísticamente significativa entre las variables con un nivel de confianza del $95.79 \%$, el modelo de segundo orden obtenido es:

Remocion de $\mathrm{OP} \%=+94.818-0.0 .117 \mathrm{H}_{2} \mathrm{O}_{2}-$ $0.622 \mathrm{Fe}(\mathrm{II})+0.267 \mathrm{H}_{2} \mathrm{O}_{2} * \mathrm{Fe}(\mathrm{II})-0.625 \mathrm{H}_{2} \mathrm{O}_{2}{ }^{2}-$ $0.796 \mathrm{Fe}(I I)^{2}(8)$

Se muestra a continuación la gráfica de efectos principales y el diagrama de Pareto:

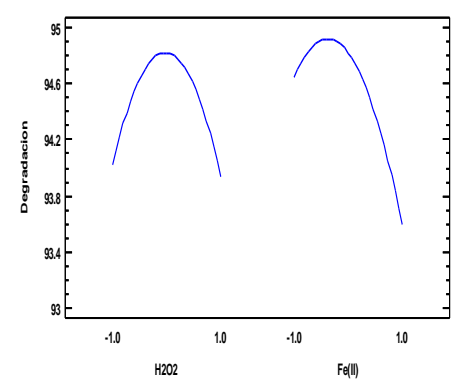

(a)

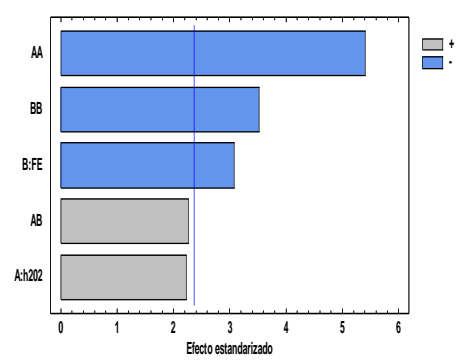

(b)
Figura 3. Grafica de los efectos principales $y$ diagrama de pareto para la remoción de la DQO.

Con el modelo cuadratico obtenido (ecuación 8), se optimizó el proceso de remoción de materia orgánica. Se muestra superficie de respuesta:
Figura 4. Grafica de contornos y superficie de

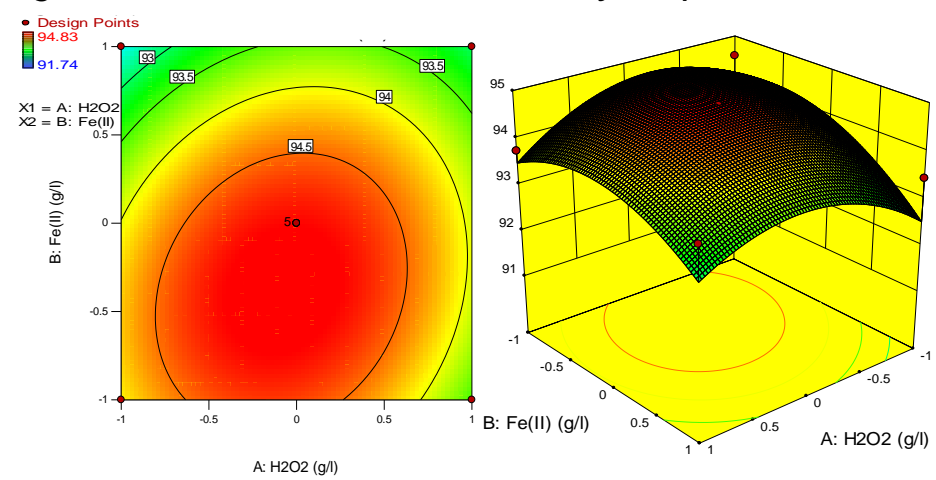

respuesta del modelo de segundo orden para la remoción de la DQO (\%).

En la Figura 4 se muestra la superficie de respuesta, se puede ver la zona donde se obtiene mayor porcentaje de remoción, con un máximo predicho por el software de $94.83 \%$, del \%DQO.

La condición experimental está dentro de dicha zona y se muestra en la Tabla 9:

Tabla 9. Dosis óptima para incrementar la remoción de $D Q O$.

\begin{tabular}{|c|c|c|c|}
\hline Factor & $\begin{array}{c}\text { Variables } \\
\text { codificadas }\end{array}$ & $\begin{array}{c}\text { Variables } \\
\text { naturales }\end{array}$ & $\begin{array}{c}\text { Remoción } \\
\text { (DQO \%) }\end{array}$ \\
\hline $\mathrm{H}_{2} \mathrm{O}_{2}$ & 0.0241 & 174.453 & $94.84 \%$ \\
\cline { 1 - 3 } $\mathrm{Fe}(\mathrm{II})$ & -0.0565 & 10.207 & \\
\hline
\end{tabular}

Haciendo uso de la ecuación (4), se convierte de variables codificadas a variables naturales, obteniendo que la condición experimental es de $74.453 \mathrm{~g} / \mathrm{L}$ de $\mathrm{H}_{2} \mathrm{O}_{2}$ y $10.207 \mathrm{~g} / \mathrm{L} \mathrm{Fe}$ (II), lo que daría como resultado $94.84 \%$ de remoción de DQO.

Con la condición experimental obtenida, se realizó un nuevo experimento y se caracterizó en base a los mismos parámetros evaluados en el agua residual.

Tabla 10. Remoción de la concentración de parámetros evaluados

\begin{tabular}{|c|c|c|c|}
\hline Parámetro & $\begin{array}{c}\text { Agua } \\
\text { residual } \\
\text { tratada }\end{array}$ & $\begin{array}{c}\text { Remoci } \\
\text { ón (\%) }\end{array}$ & VMA $^{1}$ \\
\hline $\mathrm{DQO}\left(\mathrm{mgO}_{2} / \mathrm{L}\right)^{*}$ & 495 & $95.30 \%$ & 1000 \\
\hline
\end{tabular}




\begin{tabular}{|l|c|c|c|}
\hline $\mathrm{DBO}_{5}\left(\mathrm{mgO}_{2} / \mathrm{L}\right)$ & 273 & $67.88 \%$ & 500 \\
\hline $\mathrm{pH}$ & 5.86 & 3.06 & $6-9$ \\
\hline $\begin{array}{l}\text { Fenoles } \\
\text { totales }(\mathrm{mg} / \mathrm{L})\end{array}$ & 0.002 & $100.0 \%$ & $\mathrm{NA}$ \\
\hline Nitratos(mg/L) & 7312 & $91.12 \%$ & $\mathrm{NA}$ \\
\hline Sulfatos(mg/L) & 10031 & $90.40 \%$ & 500 \\
\hline
\end{tabular}

NA: No Aplicable

1 VMA valores máximo admisibles para la descarga de aguas residuales no domesticas al sistema de alcantarillado.

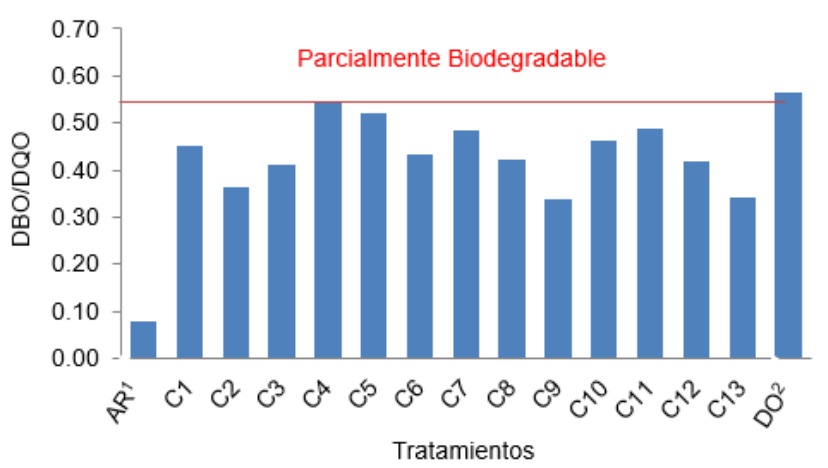

En la Tabla 10 se observa que los parámetros DQO y $\mathrm{DBO}_{5}$ se encuentran por debajo de los Valores Máximos Admisibles, mientras que en el parámetro de sulfatos, a pesar de lograr una alta remoción, no se logró reducir la concentración a valores reglamentarios de descarga a las redes de alcantarillado. Por lo que sería necesario un tratamiento posterior. En cuanto a los demás parámetros, como son los fenoles y nitratos, no están considerados en los Valores Máximos Admisibles.

\subsection{Incremento de la biodegradabilidad en aguas residuales tóxicas de los laboratorios de la UNAS}

Los resultados del incremento de la biodegradabilidad se presentan a continuación:

Tabla 11. Incremento del índice de biodegradabilidad

\begin{tabular}{|c|c|c|c|}
\hline Muestra & $\mathrm{DBO}_{5} / \mathrm{DQO}$ & Muestra & $\mathrm{DBO}_{5} / \mathrm{DQO}$ \\
\hline $\mathrm{AR}^{1}$ & 0.08 & $\mathrm{C} 8$ & 0.42 \\
\hline $\mathrm{C} 1$ & 0.45 & $\mathrm{C} 9$ & 0.34 \\
\hline $\mathrm{C} 2$ & 0.37 & $\mathrm{C} 10$ & 0.46 \\
\hline $\mathrm{C} 3$ & 0.41 & $\mathrm{C} 11$ & 0.49 \\
\hline $\mathrm{C} 4$ & 0.55 & $\mathrm{C} 12$ & 0.42 \\
\hline $\mathrm{C} 5$ & 0.52 & $\mathrm{C} 13$ & 0.34 \\
\hline $\mathrm{C} 6$ & 0.43 & $\mathrm{DO}^{2}$ & 0.57 \\
\cline { 1 - 2 } $\mathrm{C} 7$ & 0.48 & & \\
\hline
\end{tabular}

1: Agua residual

2: Dosis optima

De los resultados obtenidos del índice de biodegradabilidad, se observa un notable incremento, partiendo de un agua residual de carácter no biodegradable con un valor de 0.08 hasta el valor óptimo de 0.57 de característica parcialmente biodegradable.

Figura 5. Comparación de la biodegradabilidad y los valores obtenidos.

En la Figura 5 se puede observar que mediante los tratamientos se logró alcanzar el óptimo de biodegradabilidad con un máximo de 0.57 , indicando que el agua residual tratada es parcialmente biodegradable.

\section{4. sección de interpretación de los resultados}

De la caracterización del agua residual tóxica en la tabla 1, se puede observar que la concentración de los parámetros evaluados, superan ampliamente los Valores Máximos Admisibles de la normativa ambiental peruana usada como referencia para el desarrollo de esta investigación. Los parámetros de mayor interés son el fenol y la DQO, ya que presentaron concentraciones de $1740 \mathrm{mg} / \mathrm{L}$ y 10550 $\mathrm{mgO}_{2} / \mathrm{L}$ respectivamente. [17] manifiesta que la presencia de compuestos fenólicos se considera tóxica para algunas formas de vida acuática a partir de $0,05 \mathrm{mg} / \mathrm{L}$ (ya que se trata de compuestos con una elevada demanda de oxígeno). En esta tabla se evidencia el carácter contaminante y la necesidad de la aplicación de un tratamiento avanzado.

El agua residual estudiada fue una mezcla proveniente de diferentes laboratorios de la UNAS, con características recalcitrantes y/o toxicas, podría pensarse que tratar mezclas de contaminantes no resultaría efectiva para algún tipo de tratamiento, sin embargo [18] manifiesta que el tratar mezclas de contaminantes con el proceso Foto-Fenton resulta efectivo ya que las reacciones foto sensibilizadas de la mezcla, producen especies de oxígeno activado, así como la interacción de los complejos de hierro y las moléculas orgánicas que favorecen la efectividad del proceso, por otro lado [9] manifiesta que el proceso Fenton destaca por sus ataques no selectivos, puesto que permite la descontaminación de mezclas de contaminantes. 
De acuerdo a lo observado en la Tabla 6, el valor-P en la tabla ANVA es mayor que 0.05 , indicando que no existe una relación estadísticamente significativa entre las variables en estudio, además se obtiene que el factor $\mathrm{R}^{2}$ ajustado es cero (0) lo que indica la no existencia de relación lineal estocástica, pero no indica independencia de las variables ya que puede existir una relación no lineal incluso exacta [14], por consiguiente se puede afirmar que las condiciones experimentales se encuentran muy próximas del óptimo. En las inmediaciones del optimo, se ajustó un modelo de segundo orden, cuyo valor-P en la tabla 8 , es menor que 0.05 , indicando que existe una relación estadísticamente significativa entre las variables con un nivel de confianza del $95.79 \%$, este valor indica que el modelo cuadrático ajustado (ecuación 8) puede describir adecuadamente el comportamiento del sistema en el rango seleccionado como manifiesta [19].

En la Figura 3 (a), se muestra la gráfica de los efectos principales en la remoción de la DQO, donde se observa que inicialmente el aumento en la concentración de las variables produce una mayor remoción, pero al llegar a cierto nivel el porcentaje de remoción comienza a disminuir. Con los resultados obtenidos se coincide con [16] quien sostiene que un exceso de hierro da lugar a una disminución de la eficacia del proceso, además una baja concentración del peróxido de hidrogeno conlleva a velocidades de degradación lentas y, una adición demasiado elevada, provoca la pérdida de eficiencia en el consumo del mismo [20]. Por otro lado en la figura 3 (b) se puede observar que las interacciones de los efectos principales ( $A$ y $A B$ ) tiene significancia, el valor positivo del efecto estandarizado indica que la interacción es sinergística, es decir, que el incremento combinado de los efectos principales aumenta en mayor proporción la remoción de la DQO.

En la Figura 4 se muestra la superficie de respuesta, la que tiene característica de máxima respuesta [14], se puede ver la zona donde se obtiene mayor porcentaje de remoción, con un máximo predicho por el software de $94.83 \%$, de la DQO. La condición experimental está dentro de dicha zona y se muestra en la Tabla 9, al realizar un experimento más con la condición experimental (dosis óptima) se obtuvieron los resultados mostrados en la Tabla 10, al observar la variable respuesta (DQO) resulta 95.30\% cuyo valor es mayor al predicho por el software. Esto puede explicarse debido a que en el modelo no se consideró la temperatura como variable indirecta, y [21] sostiene que la reacción de oxidación Fenton es endotérmica, es decir aumenta con el incremento de temperatura, por lo que es una variable determinante en la eficiencia del proceso, la misma que deberá ser considerada para modelar el comportamiento del proceso Foto-Fenton en estudios posteriores.

En la Tabla 10, se puede apreciar una serie de parámetros evaluados tanto en el agua residual original como en el agua residual tratada, de los cuales en la variable DQO se logró una remoción del $95.3 \%$, para nitratos $91.12 \%$ y sulfatos en $90.40 \%$, estos resultados pueden deberse a que los procesos de oxidación avanzada pueden llevar a una oxidación de los compuestos inorgánicos hasta dióxido de carbono como sostiene [22]. La razón por la que no se alcanzó mayores porcentajes de remoción en los parámetros mencionados, puede deberse a la elevada concentración de sulfatos (104402 mg/L), ya que según [23] la elevada concentración de este anión inorgánico disminuye la velocidad del proceso, reduciendo la reactividad del Fe (III) y secuestrando radicales hidroxilo, además la presencia de Nitratos $\left(\mathrm{NO}_{3}{ }^{-}\right)$da lugar a la formación de complejos insolubles con el Fe (III).

Otro de los parámetros evaluados es el fenol, [24] manifiesta que el fenol al combinarse con el cloro da lugar a clorofenoles, compuestos altamente tóxicos, cancerígenos y muy difíciles de eliminar, sin embargo en el presente estudio al aplicar el tratamiento con Foto-Fenton se logró remover el $100 \%$ de fenol contenido en agua residual tóxica, con el resultado obtenido se coincide con [17], quien sostiene que los fenoles pueden ser degradados fácilmente por la tecnología de Foto-Fenton.

De los resultados obtenidos del índice de biodegradabilidad, se observa un notable incremento, partiendo de un agua residual de carácter no biodegradable con un valor de 0.08 hasta el valor óptimo de 0.57 de característica parcialmente biodegradable, con este resultado se coincide con [25], quien manifiesta que con la aplicación del proceso Foto-Fenton se logra un aumento de la biodegradabilidad del agua residual y la transformación de compuestos tóxicos y/o inhibidores en compuestos menos nocivos.

En la Figura 5 se puede observar que mediante los tratamientos se logró alcanzar el óptimo de biodegradabilidad con un máximo de 0.57, indicando que el agua residual tratada es parcialmente biodegradable. La razón por la que no se alcanzó un valor fácilmente biodegradable (índice de biodegradabilidad > 0.59) puede verse influenciado por las reacciones químicas que 
ocurren durante el proceso, un ejemplo claro viene a ser la degradación del fenol, ya que según [25], se presentan los siguientes compuestos intermediarios de forma secuencial: anillos dihidroxilados, benzoquinonas y anillos aromáticos, una vez que se rompe el anillo aromático, aparecen los hidrocarburos ácidos y finalmente, los ácidos orgánicos de cadena más corta. Estos ácidos de cadena corta son: acético, oxálico, fórmico, maleico y fumárico, los cuales son identificados como resistentes a la reacción fenton como lo menciona [26].

\section{Conclusiones}

Se caracterizó las aguas residuales tóxicas provenientes de los laboratorios de la Universidad Nacional Agraria de la Selva, obteniendo valores de (DQO $10550 \mathrm{mgO} / \mathrm{L}, \mathrm{DBO}_{5} 859 \mathrm{mgO}_{2} / \mathrm{L}$, fenoles totales $1740 \mathrm{mg} / \mathrm{L}$, sulfatos $104402 \mathrm{mg} / \mathrm{L}$, nitratos $83250 \mathrm{mg} / \mathrm{L} \mathrm{y} \mathrm{pH} 2.8$ ), cuyas concentraciones superan ampliamente los Valores Máximos Admisibles.

Se logró optimizar el proceso Foto-Fenton (dosis $\mathrm{H}_{2} \mathrm{O}_{2}$ y $\mathrm{Fe}$ II) a través de la metodología de superficie de respuesta, cuya concentración de Fe (II) fue la variable significativa sobre proceso. La dosis optima con la que se consigue valores de remoción de $95.3 \%$ de la $\mathrm{DQO}, 92.8 \%$, de nitratos, $90.4 \%$ sulfatos y un $100 \%$ de fenoles totales, fue $174.453 \mathrm{~g} / \mathrm{L}$ de $\mathrm{H}_{2} \mathrm{O}_{2}$ y $10.207 \mathrm{~g} / \mathrm{L}$ de $\mathrm{Fe}$ (II).

Se incrementó el índice de biodegradabilidad, partiendo de un valor 0.08 de contenido de materia orgánica difícilmente biodegradable, hasta un óptimo de 0.57 de contenido de materia orgánica parcialmente biodegradable.

\section{Agradecimientos}

Para realizar esta investigación se contó con la asesoría del Ing. M.S.c. José Antonio Blas Matienzo, Ing. M.S.c. José Luis paredes Salazar y el Dr. Emel López Villanueva. A quienes estoy agradecido por sus oportunas sugerencias.

\section{Referencias}

[1] C. Colmenares, M. Bases para el manejo de sustancias químicas peligrosas, en laboratorios de docencia de la escuela de ingeniería química, Universidad de Carabobo. Centro de investigaciones ambientales de la Universidad de Carabobo, (2014) 31-32.

[2] B.A. Castro, B., V.A. Huetio, A.C. Mera. Fotocatálisis Heterogénea con $\mathrm{TiO}_{2} \mathrm{y}$
$\mathrm{H}_{2} \mathrm{O}_{2}$ para el tratamiento de desechos líquidos con presencia de Fenolftaleína generados en los laboratorios de análisis químico de la Universidad del Cauca. Universidad del Cauca (2008) 17-19.

[3]. E. Ramírez, P. Mijaylova, J. Cruz, M. Rodriguez. Aporte de diferentes mecanismos en la remoción de sustancias orgánicas tóxicas presentes en aguas residuales industriales. XXVII Congreso Internacional de Engenharia Sanitaria e Ambiental. Scielo (2010) 2-3.

[4] K.Ramos, Y. Jimenez. Degradación de desechos tóxicos provenientes de laboratorios universitarios con foto-fenton solar usando diseño de experimentos. Redalyc, 32 (2016) 119-131.

[5] J.C. García, M.P. Castellanos, M., Uscátegui, J. Fernández, A.M. Pedroza, C.E. Daza. Remoción de colorantes sintéticos mediante el proceso Fenton heterogéneo usando $\mathrm{Fe}_{2} \mathrm{O}_{3}$ soportado en carbón activado obtenido a partir de residuos de rosas. Univ. Sci. 17 (3) (2012) 303-314.

[6] V.A, Sakkas., Md. Azharul, C. Stalikas C. Y T.A. Albanis. Photocatalytic degradation using design of experiments: Journal of Hazardous Materials, 175 (2010) 33-34.

[7] J. Blanco, S. Malato, J. Peral, B. Sánchez, A.I. Cardona. Diseño de reactores para fotocatálisis. Sol. Energy (2008) 19-21.

[8] M. Chong, N. Jin, C.Chow, Y C. Saint, Recent developments in photocatalytic water treatment technology. Sol. Energy (2010) 2997-3027

[9] S. Malato, I.P. Fernández, M. I. Maldonado, J. Blanco, W.Gernjak. Decontamination and desinfection of water by solar photocatalysis. Sol. Energy (2009) 513524.

[10] S.Kenfack, C. Pulgarin. Helio-photoFenton enhancement of the biodegradability of biorecalcitrant wastewaters: Physico-chemical and technical aspects. Tesis doctoral, Ecole Polytechnique Fédérale de Lausanne, Lausanne, Suiza, 2006.

[11] APHA-AWWA-WEF. Standard Methods for the Examination of Water and Wastewater. 22 Ed. (American Public Health Association/ American Water Works Association/Water Environment Federation, Washington, 2012), pp. 984. 
[12] EPA Method. Environmental protection agency methodos for chemical analysis of wastes. Environmental Protection Agency (1963), pp. 430.

[13] J.C. Rojas. Potabilización de aguas superficiales mediante proceso de ultrafiltración con membranas arrolladas en espiral (Universidad de granada 2008), p 271.

[14] C. Montgomery, Control estadístico de la calidad. 3ra ed. (Limusa WILEY, 2001), pp. 791.

[15] P. Cisterna. Determinación de la relación $\mathrm{DBO}_{5} / \mathrm{DQO}$ en las aguas residuales de comunas con población menor a 25000 habitantes en la VIII región. Universidad Tec. Fed. Sta. María (2010) 10-11.

[16] J. Blanco. Degradación de un efluente textil real mediante Fenton y Foto-Fenton. Tesis de master, Universitat politécnica de Catalunya, 2009.

[17] L.A. Aznate, O.J. Cerro, A.Orozco. Degradación por fotocatálisis homogénea (foto-fenton) de efluentes líquidos contaminados con residuos de fenol. Tesis de pregrado, Universidad de Cartagena, 2013.

[18] M. Lapertot, S, Ebrahimi, S. Dazio, A. Rubinelli, C. Pulgarin. Photo-Fenton and biological integrated process for degradation of mixture of pesticidas. Journal of Hazardous Materials, 186 (2007) 34-40.

[19] B. Bianco, I. De Michelis, F.Vegliò. Fenton treatment of complex industrial wastewater: Optimization of process conditions by surface response method. Journal of hazardous material, 186 (2011) 1733-1738.

[20] R.A. Cabrera. Combinación de Fotocatálisis solar con Biorreactores de Membrana para el Tratamiento de Aguas Tóxicas. Modelado del proceso Foto Fenton como Herramienta de Diseño y Optimización. Dr. Tesis, Universidad de Almeria, 2013.
[21] H. Zhang, H.J Choi, C. Huang. Optimization of Fenton process for the treatment of landfill leachate. Journal of Hazardous Materials, 125 (2005) 166-174.

[22] M.I. Castellar, J.C.Osorio Tamayo. Estado del arte de la fotocatálisis como técnica para remoción de efluentes químicos provenientes de laboratorios. Tesis de pre grado, Universidad de San Buenaventura, 2012.

[23] J. Pignatello, E. Oliveros, A. Mackay. Advanced Oxidation Processes for organic contaminant destruction based on the Fenton reaction and related chemistry. Environmental Science \& Technology, 26 (2006) 35, 1-84.

[24] M. Rodriguez. Fenton and UV-vis based advanced oxidation processes in wastewater treatment: Degradation, mineralization and biodegradability enhancement. Tesis Doctoral, Universidad de Barcelona, 2003

[25] X. Wang, S. Chen, X. Gu, K. Wang. Pilot study on the advanced treatment of landfill leachate using combined coagulation, fenton oxidation and biological aerated filter process. Journal of Hazardous Materials, 29 (2009) 1354-1358.

[26] F. Mijingos, F. Varona, N. Violeta Changes in solution color during phenol oxidation by Fenton reagent. Enviromental Science \& Technology, 40 (2006) 5538-5543.

Autor correspondiente:

gianastillo1994@yahoo.com 